\title{
Performance and characterization of a modular superconducting nanowire single photon detector system for space-to-Earth optical communications links
}

\author{
Brian E. Vyhnalek, Sarah A. Tedder, and Jennifer M. Nappier \\ National Aeronautics and Space Administration \\ Glenn Research Center \\ Cleveland, OH, USA
}

\begin{abstract}
Space-to-ground photon-counting optical communication links supporting high data rates over large distances require enhanced ground receiver sensitivity in order to reduce the mass and power burden on the spacecraft transmitter. Superconducting nanowire single-photon detectors (SNSPDs) have been demonstrated to offer superior performance in detection efficiency, timing resolution, and count rates over semiconductor photodetectors, and are a suitable technology for high photon efficiency links. Recently photon detectors based on superconducting nanowires have become commercially available, and we have assessed the characteristics and performance of one such commercial system as a candidate for potential utilization in ground receiver designs. The SNSPD system features independent channels which can be added modularly. We analyze the scalability of the system to support different data rates, as well as consider coupling concepts and issues as the number of channels increases.
\end{abstract}

Keywords: Optical communications, single photon detectors, superconducting nanowire

\section{INTRODUCTION}

Free-space optical communications (FSOC) is an extremely promising solution for higher-rate data communications from lunar and inter-planetary distances to Earth-based ground stations, offering many advantages as compared to radio-frequency (RF) technologies such as the potential for substantially higher data rates and lower mass, power and size. NASA's 2013 Lunar Laser Communication Demonstration (LLCD) showed that FSOC from the Moon to Earth is feasible and advantageous by achieving up to $622 \mathrm{Mbps}$ on the optical downlink, substantially exceeding the fastest Ka-band RF links. ${ }^{1}$ With NASA's ongoing efforts such as the Jet Propulsion Laboratory's (JPL) Deep Space Optical Communications (DSOC) project, ${ }^{2}$ the Laser Communication Relay Demonstration (LCRD), ${ }^{3}$ and the Optical-to-Orion $(\mathrm{O} 2 \mathrm{O})$ project, the future prospects continue to improve for mission-operational free-space optical communications.

A number of enabling technologies were demonstrated in the LLCD mission including the use of multi-element niobium nitride $(\mathrm{NbN})$ and multi-element tungsten silicide (WSi) superconducting nanowire single-photon detector (SNSPD) arrays developed by the Massachusetts Institute of Technology's Lincoln Laboratory and JPL, respectively, and used at the LLCD ground stations in White Sands, New Mexico and on top of Table Mountain in California. ${ }^{1}$ SNSPDs have been under investigation since the early $2000 \mathrm{~s},{ }^{4}$ and have shown great promise for a variety of ultra-sensitive light detection applications - in particular for applications at telecommunications wavelengths near $1550 \mathrm{~nm}$ where they substantially outperform single-photon InGaAs avalanche photo-diodes (APDs) and photomultiplier tubes (PMTs). ${ }^{5-7}$ Until very recently photon detectors based on SNSPDs were not commercially available, thus limiting widespread usage. With several commercial vendors offering turnkey multichannel SNSPD systems with simplified cryogenics, SNSPDs are increasingly viable as an operational component for space-to-ground high-photon efficiency FSOC links.

\footnotetext{
Send correspondence to brian.e.vyhnalek@nasa.gov
} 


\section{DETECTOR SYSTEM CHARACTERIZATION}

We characterized a two-detector Opus One ${ }^{T M}$ system (Figure 1) from Quantum Opus, LLC which operates at a temperature of $2.5 \mathrm{~K}$ using a closed-cycle helium water-cooled cryocooler housed in a 3U 19-inch rack-mountable unit, and is modularly exapandable to up to 16 detectors onto a single cold head. The nanowire detectors are optimized for $1550 \mathrm{~nm}$ operating wavelength, self-aligned for maximum coupling efficiency, ${ }^{8,9}$ and coupled to single-mode fiber inputs. Electrically the devices are current biased with adjustable front panel controls, and coupled to $50 \Omega$ coaxial readout cables to room temperature amplifiers with $500 \mathrm{MHz}$ bandwidth and a maximum 55.6 dB gain. ${ }^{10}$ The system is powered via a Standford Research Systems SIM900 mainframe, and can be computer controlled either serially or through GPIB interface.

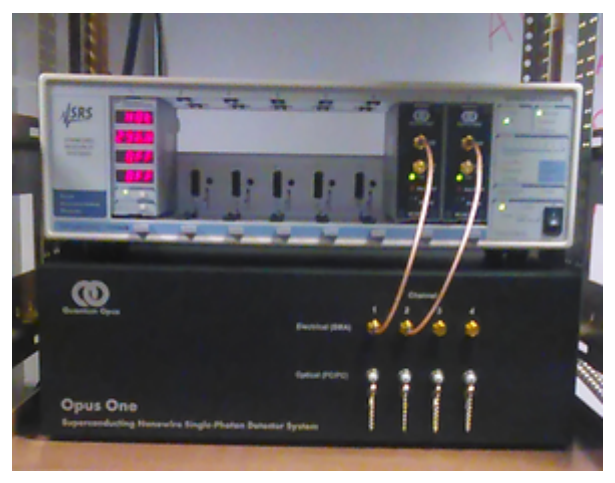

Figure 1: Opus One ${ }^{T M}$ SNSPD system.

The system detection efficiency (SDE) and dead-time characterization was measured using the setup shown in Figure 2. A benchtop continuous wave (CW) distributed feedback (DFB) laser source was thermally controlled to output precisely at an operating wavelength of $1550 \mathrm{~nm}$, and attenuated to a mean photon number of $\approx 168,000$ photons/s using both fixed and variable attenuators. Each component in the setup was separately characterized

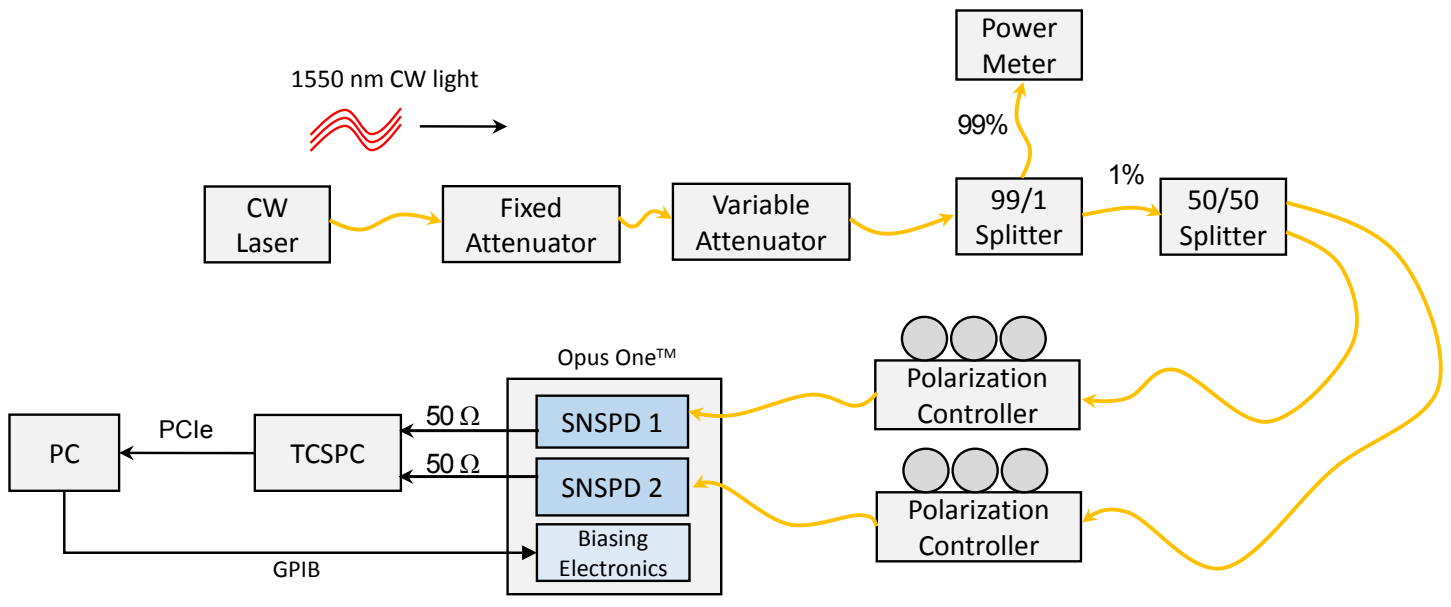

Figure 2: Characterization setup diagram

with a calibrated $1550 \mathrm{~nm}$ InGaAs power meter with $0.1 \mathrm{pW}$ resolution to determine fiber loss, fiber splitting ratios, and attenuation factors before being assembled together with a tap to the power meter to monitor input power. Fiber paddle polarization controllers were placed before the inputs to the fiber coupling to the SNSPDs to adjust the input state of polarization in order to accommodate the polarization sensitivity of the detectors. The ouput pulses from the SNSPDs were sent to a multi-channel time-correlated single-photon counting (TCSPC) card, capable of 25 ps resolution and up to 50 Mcps continuous counting per channel. 
Dark count rate and background counts were initially determined for each detector channel as a function of bias current as shown in Figure 3a, where each data point is the average of 10 measurements. The dark count rates were measured with input fibers to the system disconnected and the inputs to the detectors capped, whereas the background count rates were determined with the input fibers connected, but with the laser blocked by a shutter. Each channel displayed about the same level of dark count rate, less than $10-30$ cps for bias currents $I_{B}<15 \mu \mathrm{A}$, and less than $100 \mathrm{cps}$ for $15<I_{B} \lesssim 15.5 \mu \mathrm{A}$ before increasing by several orders of magnitude between 15 and $I_{S W} \approx 16 \mu \mathrm{A}$, the level of input current at which the devices switch from the superconducting to the normal state. Background count rates were nearly constant and identical for both channels at a level of about $6 \mathrm{kcps}$, almost entirely due to room-temperature blackbody radiation except for at bias levels approaching $I_{S W}$ in which dark counts increase significantly.

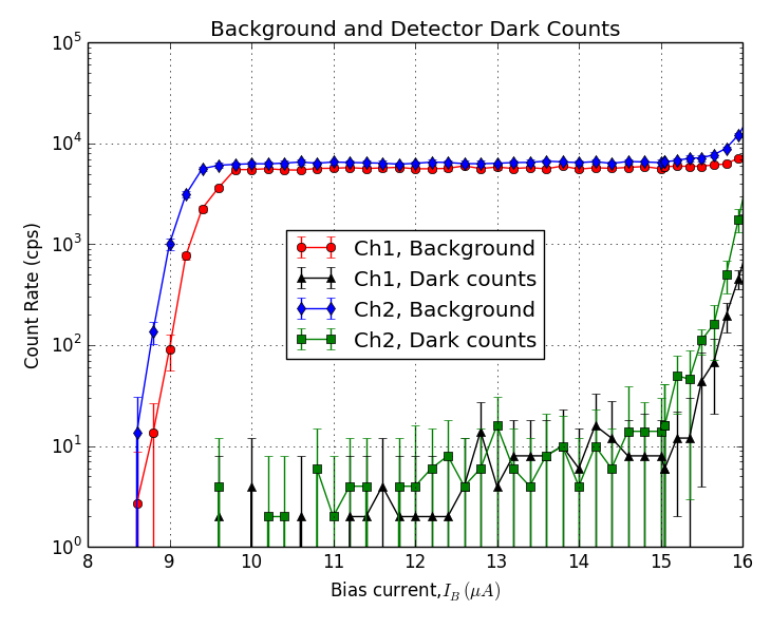

(a)

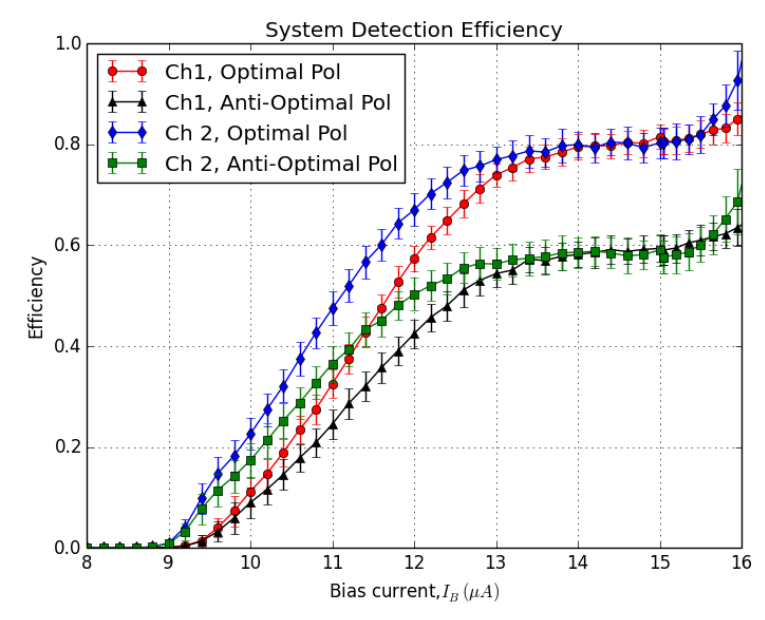

(b)

Figure 3: Background count rate and system detection efficiency vs. bias current.

System detection efficiency (SDE) was estimated for each channel for both optimal and anti-optimal input polarizations. Figure 3b shows the average and one standard deviation of 10 measurements for each bias current. The system detection efficiency was determined by $S D E=\left(R_{\text {out }}-B C R\right) / R_{\text {in }}$, where $R_{\text {out }}$ is the measured output count rate, $B C R$ is the background count rate measured previously, and $R_{i n}$ is the input photon flux estimated from power meter measurements and accounting for system losses. For both channels the SDE for optimal input polarization $\left(\mathrm{SDE}_{\max }\right)$ reached a plateau value of $80 \pm 2.8 \%$ for bias currents of $\approx 13.5-15.5 \mu \mathrm{A}\left(0.84 I_{S W}-\right.$ $\left.0.97 I_{S W}\right)$ and a maximum value of $82 \pm 2.8 \%$ for channel 1 and $82 \pm 3.1 \%$ for channel 2 at $I_{B}=0.97 I_{S W}$, after which dark counts increase significantly above $100 \mathrm{cps}$. With the polarization controllers adjusted such that the input state of polarization was orthogonal to the optimal state, a plateau value of $\approx 60 \pm 3.0 \%\left(\mathrm{SDE}_{\min }\right)$ for the same bias current range, and a maximum value of $61.0 \pm 3.0 \%$ for channel 1 and $60.2 \pm 2.9 \%$ for channel 2 at $I_{B}=0.97 I_{S W}$. The polarization dependence ratio, $r_{p o l}=S D E_{\max } / S D E_{\max }$, is determined to be a factor of $\approx$ $1.25 \mathrm{~dB}$.

A typical output pulse is shown in Figure $4 \mathrm{a}$, at a bias level of $I_{B} \approx 15 \mu \mathrm{A}$. The pulse height is $588 \mathrm{mV}$, but can range between $\approx 300 \mathrm{mV}-600 \mathrm{mV}$ depending on $I_{B}$. Rise time is $1 \mathrm{~ns}$, and the $90 \%-10 \%$ fall time of the pulse is $35 \mathrm{~ns}$, while the $1 / e$ fall time is $\tau_{r} \approx 16 \mathrm{~ns}$, faster than the values reported for WSi or molybdenum silicide (MoSi) SNSPDs ${ }^{11,12}$ but slower than values reported for $\mathrm{Nb}$ and $\mathrm{NbN}$ devices. ${ }^{4-6,13}$ Figure $4 \mathrm{~b}$ shows the histogram of the time interval for subsequent pulses, peaking at $22.5 \mathrm{~ns}$, and with no counts registered for interarrival times less than $12 \mathrm{~ns}$. The count rate as a function of the input photon flux is shown in Figure 5 with a linear fit to the first 6 data points, and a Monte Carlo simulation curve based on a phenomenological exponential recovery model of detection efficiency, i.e.

$$
S D E(t)=S D E_{\max }\left(1-e^{-\left(t-t_{0}\right) / \tau_{r}}\right)
$$




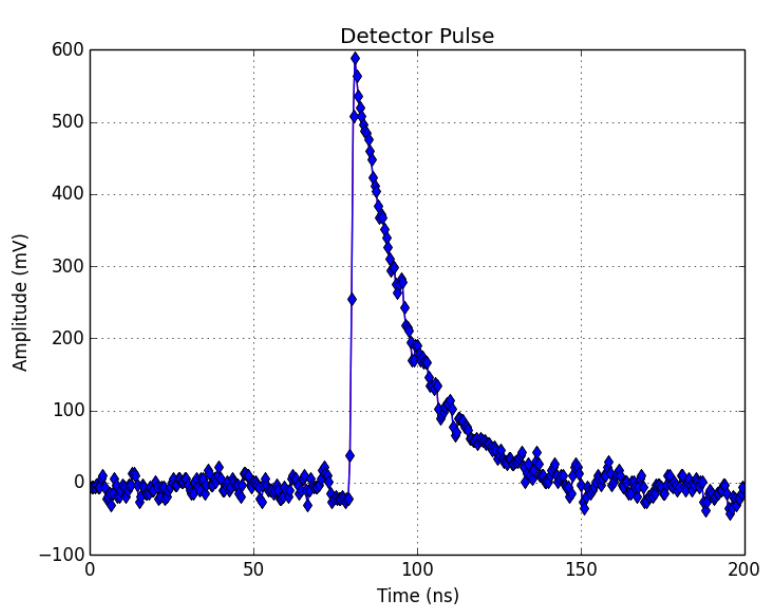

(a)

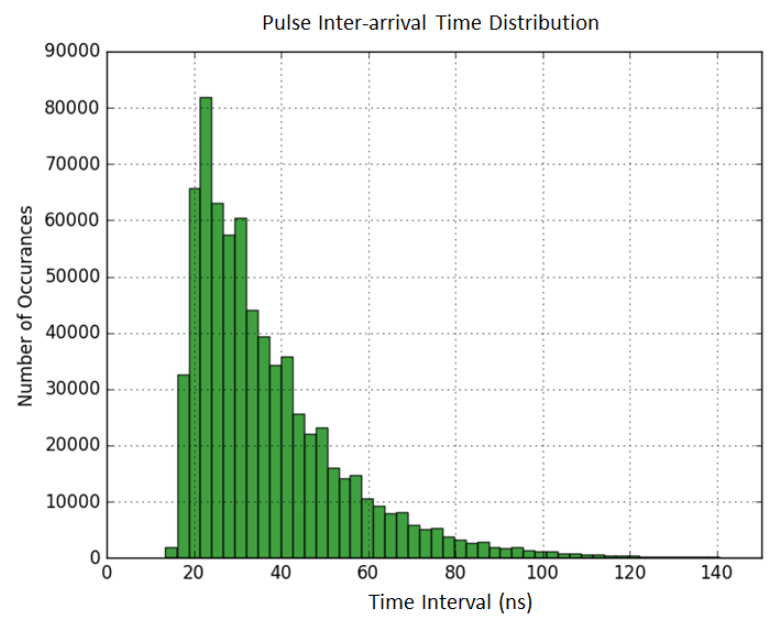

(b)

Figure 4: Example output pulse and inter-arrival time histogram.

where $S D E_{\max }$ is the maximum system detection efficiency, i.e. $82 \%, \tau_{r}$ is the $1 / e$ pulse decay time, and $t_{0}$ is a Poisson-distributed photon arrival time. From Figure 5 it can be seen that the measured counts/s increase linearly until about 12 Mcps afterwhich begin to plateau approaching a maximum of $\approx 48 \mathrm{Mcps}$, although at severly degraded detection levels approaching $4 \%$ efficiency.

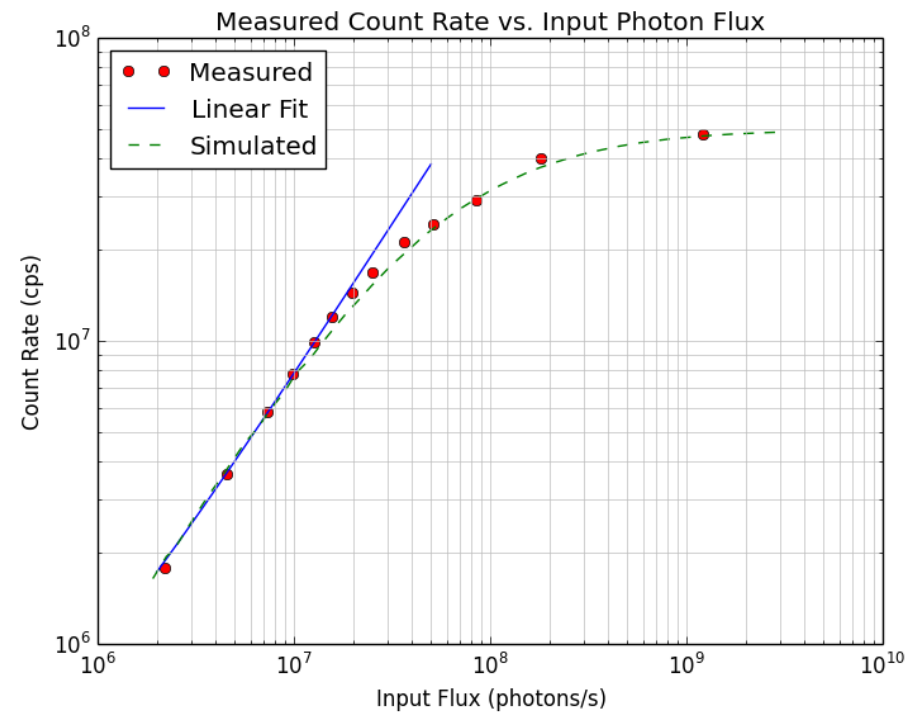

Figure 5: Measured count rate vs. estimated input photon flux for one detector.

To characterize the system instrument response function (IRF) and detection jitter, the setup was modified as shown in Figure 6 to include a $1550 \mathrm{~nm}$ femtosecond fiber laser in place of the CW DFB laser. The femtosecond laser output pulses with a minimum of $100 \mathrm{fs}$ FWHM, and was attenuated to a level of $\ll 1$ photon/pulse. An electrical pulse was also simultaneously output from the laser synchronized with the rising edge of the optical pulse, and was fed into the synchronization input of the TCSPC card. The TCSPC card then measured the time interval between the rising edge of the electrical sync pulse from the laser and the rising edge of the output 
pulse from the SNSPDs, and built up a statistical distribution with 25 ps bins. Figure 7 shows measured IRFs for channel 1 at different bias currents.

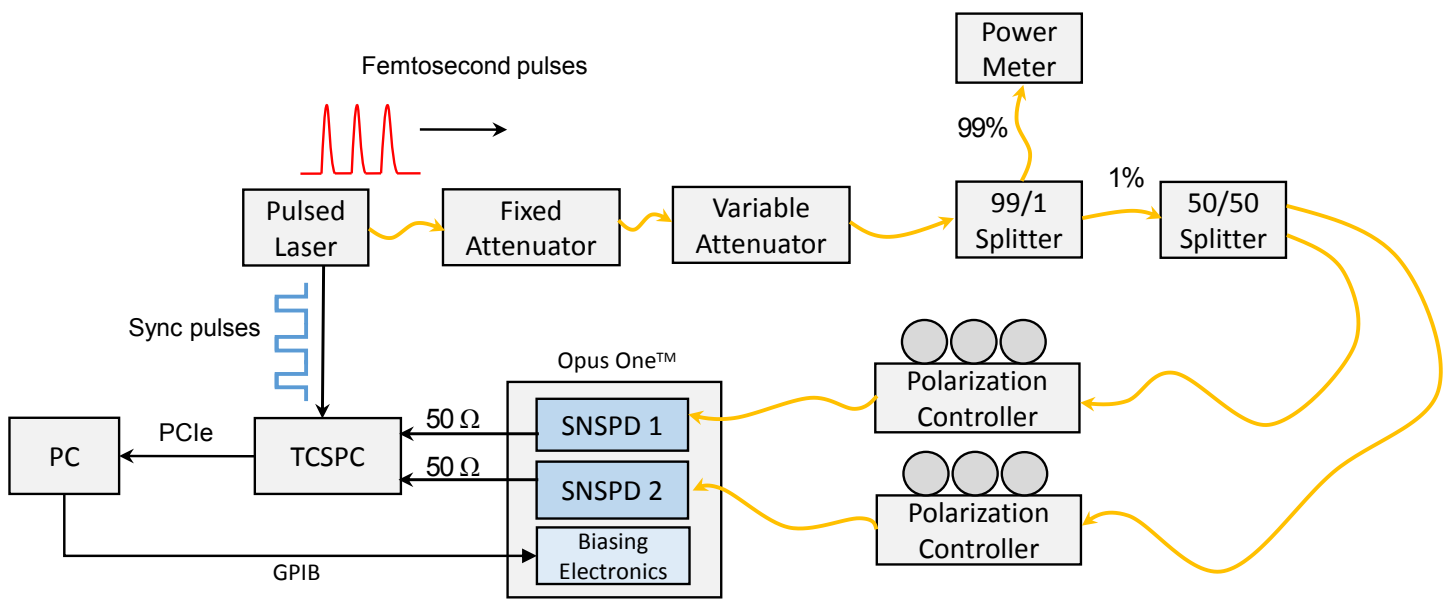

Figure 6: IRF/jitter characterization setup diagram.

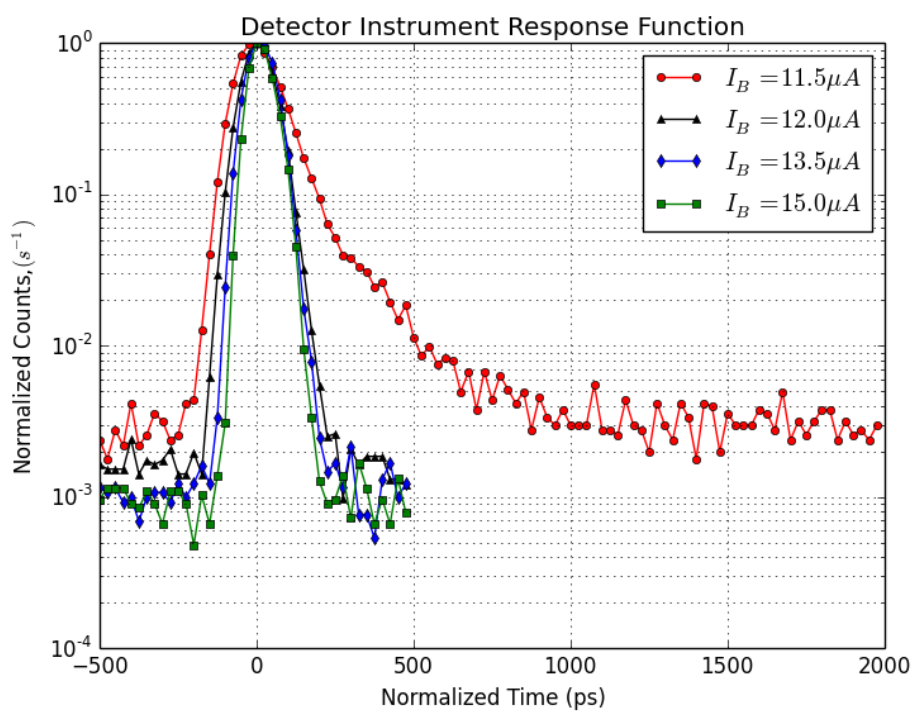

Figure 7: Example measured instrument response functions.

For each of the measured IRFs we fit a Gaussian function as shown in Figure 8a, and from the fitted functions extracted the total measurement jitter, defined as the FWHM of the IRF. ${ }^{12}$ However, these values also include jitter effects from the input laser, input sync pulse, and TCSPC card, thus the system jitter $J_{S}$ can be determined from $^{12}$

$$
J_{\text {meas }}^{2}=J_{\text {laser }}^{2}+J_{\text {sync }}^{2}+J_{T C S P C}^{2}+J_{S}^{2} .
$$

With $J_{\text {laser }}=0.06 \mathrm{ps}, J_{\text {sync }}=4.0 \mathrm{ps}$, and $J_{T C S P C}=20.0 \mathrm{ps}, J_{S}$ was calculated for each channel, and the dependence on bias current $I_{B}$ is shown in Figure 8b. From Figure 7 it can be seen that the width of the IRF decreases with increasing $I_{B}$, and similarly Figure $8 \mathrm{~b}$ shows that $J_{S}$ decreases from $\approx 110 \mathrm{ps}-120 \mathrm{ps}$ at $I_{B}=0.75 I_{S W}$ to $\approx 80-85 \mathrm{ps}$ at $I_{B}=0.97 I_{S W}$. The fact that the system jitter decreases with increasing $I_{B}$ is consistent with the fact that at higher $I_{B}$ the output pulse amplitudes are larger, and therefore higher signal-to-noise ratio. ${ }^{11}$ 


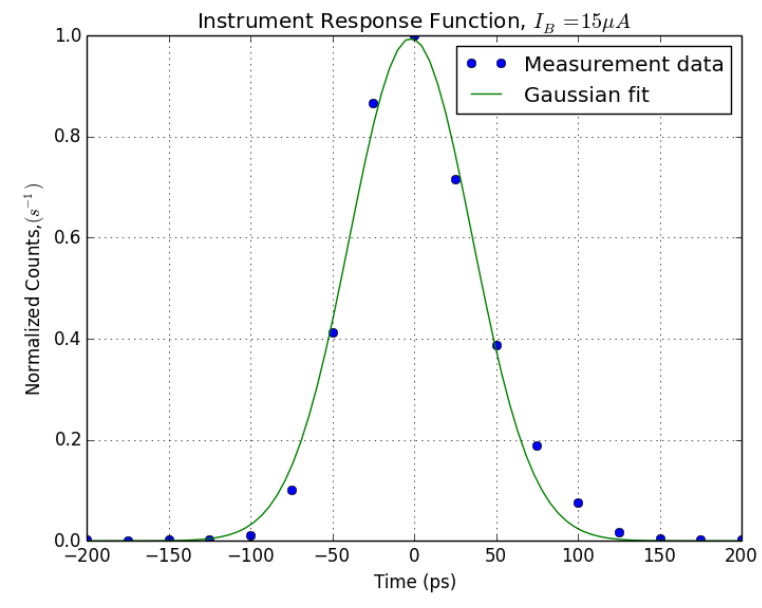

(a)

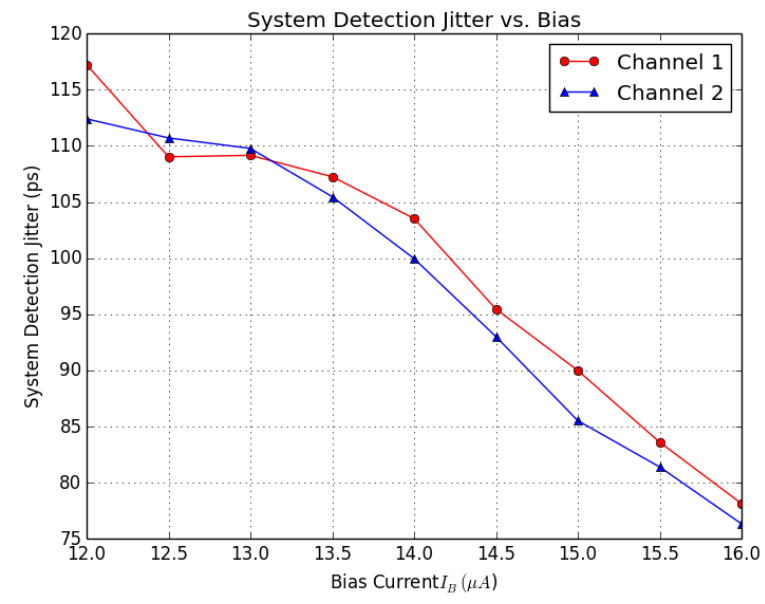

(b)

Figure 8: Example IRF with Gaussian fit and system jitter vs. bias current $I_{B}$.

\section{DEMONSTRATION IN AN OPTICAL COMMUNICATIONS LINK}

To demonstrate the utility of the commercial off-the-shelf (COTS) SNSPD system in a communications link, we perform optical link testing with the setup shown in Figure 9. The additions from the characterization setup were a polarization-adjustable $1550 \mathrm{~nm} \mathrm{CW}$ laser source with a polarization extinction ratio of up to $40 \mathrm{~dB}$, a high extinction-ratio $\mathrm{LiNbO}_{3}$ electro-optic modulator capable of over $50 \mathrm{~dB}$ of intensity extinction, and a fiber-coupled tungsten-halogen broadband light source with a $1550 \pm 20 \mathrm{~nm}$ bandpass filter along with an additional variable attenuator to control input background noise levels. The input to the modulator was

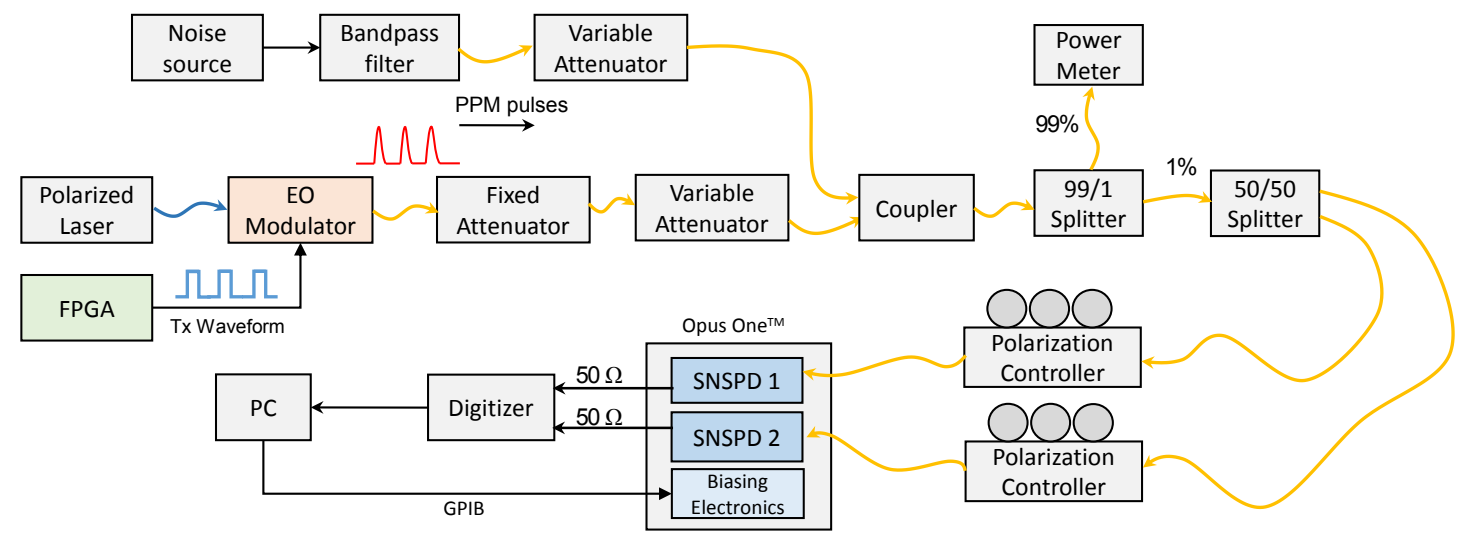

Figure 9: Laboratory test setup for optical link testing.

a serially-concatenated pulse-position modulated (SCPPM ${ }^{14}$ signal generated from a field programmable gate array (FPGA) with selectable code rates $r=\{1 / 3,1 / 2,2 / 3\}$, PPM orders $M=\{4,8,16,32,64,128,256\}$, and adjustable slot width $T_{s}$, consistent with the Consultative Committee for Space Data Systems (CCSDS) optical communications high photon efficiency (HPE) downlink transmit waveform modes. ${ }^{15,16}$ For the purposes of the demonstration we selected $M=32$, with 8 guard slots, $r=1 / 3$, and $T_{s}=4$ and $2 \mathrm{~ns}$, corresponding to data rates of 10 and $20 \mathrm{Mbps}$ respectively. Additionally, the noise source attenuation level was set such as to consistently produce an average detected background count rate of $K_{b} \approx 0.01$ counts per slot. Both the modulated $1550 \mathrm{~nm}$ optical signal and the filtered broadband noise source were combined before being input to the SNSPD detectors, and the output signals were sampled with a high-speed digitizer at a rate of $1 \mathrm{GS} / \mathrm{s}$ before being post processed. 


\section{RESULTS}

The optical link was successfully closed error-free (zero bit errors) at both the 10 Mbps and 20 Mbps rates using the output from only a single detector, with a predicted average number of detected signal counts per PPM symbol of $K_{s} \approx 0.8$. Combining the two channels together resulted in nearly double detected signal counts per PPM symbol as anticipated. Based on these results and simulation results such as those shown in Figures 10a and $10 \mathrm{~b}$, we expect that $40 \mathrm{Mbps}$ rates $\left(T_{s}=1 \mathrm{~ns}\right)$ are possible with a single detector in low background conditions, and that $80 \mathrm{Mbps}\left(T_{s}=0.5 \mathrm{~ns}\right)$ data rates are achievable using at least 2 detectors combined. These simulations

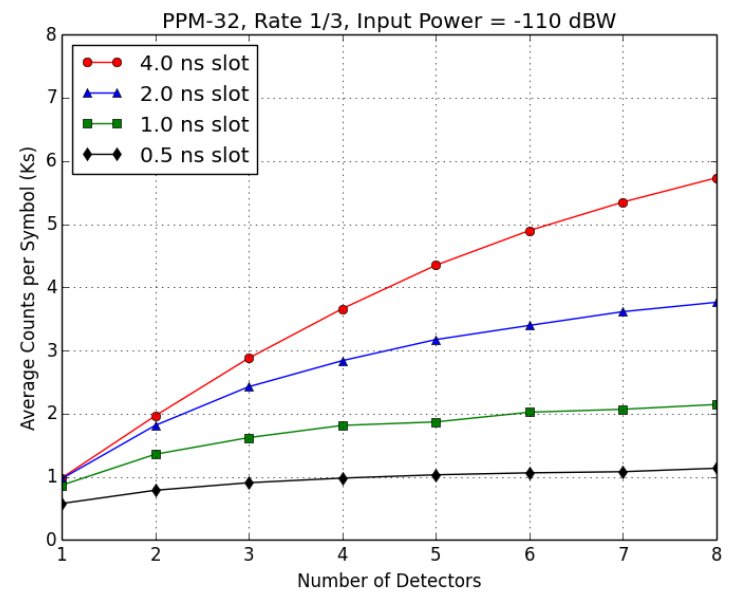

(a)

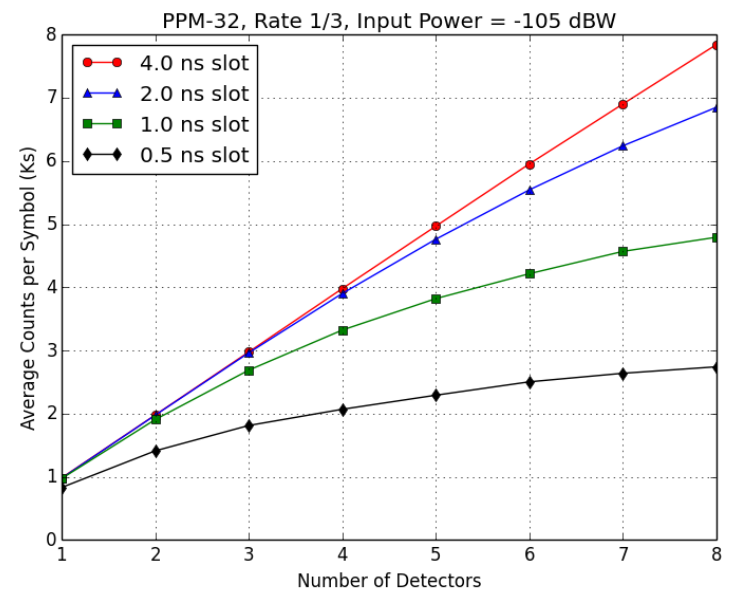

(b)

Figure 10: Simulated average number of detected signal counts per PPM symbol vs. number of detectors for PPM-32 rate 1/3 with 8 guard slots, for fixed signal and background photon flux.

were performed using the measured detector efficiency and dead time parameters, and by assigning a randomly generated Poisson-distributed photon number and arrival times within either a PPM signal slot or a background slot. The average photon number per slot was determined from the average input signal and background optical power, which for Figures 10a and 10b were -110.0, -121.2 dBW and -105.0, -121.2 dBW respectively. In this case, the background power level of $-121.2 \mathrm{dBW}$ was selected to produce an average background level of $K_{b} \approx 0.01$ counts per slot. A photon was considered to be detected if a uniformly-distributed random number over the interval $[0,1]$ was less then the $S D E$ at a photon arrival time $t$, and detector blocking effects were accounted for by allowing the detector $S D E(t)$ to follow the exponential recovery model of Eq. 1. Each successful photon detection was then registered as a signal count if the arrival/detection time was within a signal slot, otherwise as a background count.

The required number of detectors for a target data rate is a multidimensional problem depending on the modulation order, code rate, background power and expected signal power. However, because detectors plus associated control and amplifier electronics can be added modularly with minimal downtime, an SNSPD system such as this could support a variety of different optical communications links requiring single-photon sensitivity. The modular structure appropriately lends itself to receiver array architectures, but also for single aperture receivers as well. In this case losses from splitting in order to couple to multiple detectors will have to be carefully considered, and more efficient low-loss interfaces such as photonic lanterns ${ }^{17}$ may need to be further developed.

\section{CONCLUSION}

We characterized a COTS superconductor nanowire single-photon detector system to assess parameters such as detection efficiency, dark count rate, reset time, maximum count rate, and timing jitter to determine suitability 
of the current state of such systems to support upcoming and future space-to-ground missions requiring singlephoton sensitivity. The measured SNSPD parameters were similar to the results reported for $\mathrm{WSi}^{12}$ and $\mathrm{MoSi}^{11}$ in terms of detection efficiency and detection jitter, and exceeded the results reported for count rate and reset time for single-element devices in those materials. We successfully closed an optical communications link with zero bit errors, and have shown that an error-free $20 \mathrm{Mbps}$ data rate is possible with a single detector while anticipating that $80 \mathrm{Mbps}$ may be possible with two detectors. As SNSPD technology continues to mature continual improvements in sensitivity, reset time and jitter are anticipated. Along with the ability to add detectors modularly in parallel, thereby adding a degree of flexibility to system design, the prospects for optical communications used operationally in lunar and inter-planetary space missions continue to improve.

\section{ACKNOWLEDGMENTS}

The authors would like to acknowledge the support by the NASA Space Communications and Navigation (SCaN) funded Integrated Radio and Optical Communications (iROC) project, and Aaron Miller and Tim Rambo from Quantum Opus LLC for useful discussions.

\section{REFERENCES}

1. B. S. Robinson, D. M. Boroson, D. A. Burianek, D. V. Murphy, F. I. Khatri, J. W. Burnside, J. E. Kansky, A. Biswas, Z. Sodnik, and D. M. Cornwell, "The NASA Lunar Laser Communication Demonstration successful high-rate laser communications to and from the Moon," in Proceedings of SpaceOps, 2014.

2. H. Hemmati, A. Biswas, and I. Djordevic, "Deep Space Optical Communications: Future perspectives and applications," in Proceedings of the IEEE, 99(11), pp. 2020 - 2039, 2011.

3. B. L. Edwards, D. Israel, K. Wilson, J. Moores, and A. Fletcher, "Overview of the laser communications relay demonstration project," in Proceedings of SpaceOps, pp. 11 - 15, 2012.

4. R. Sobolewski, A. Verevkin, G. N. Gol'tsman, A. Lipatov, and K. Wilsher, "Ultrafast superconducting single-photon optical detectors and their applications," IEEE Transactions on Applied Superconductivity 13, pp. 1151-1157, June 2003.

5. A. J. Kerman, E. A. Dauler, B. S. Robinson, R. Barron, D. O. Caplan, M. L. Stevens, J. J. Carney, S. A. Hamilton, W. E. Keicher, J. K. W. Yang, K. Rosfjord, V. Anant, and K. K. Berggren, "Superconducting nanowire photon-counting detectors for optical communications," Lincoln Laboratory Journal 16(1), pp. 217-224, 2006.

6. C. M. Natarajan, M. G. Tanner, and R. H. Hadfield, "Superconducting nanowire single-photon detectors physics and applications," Superconductor Science and Technology 25(6), 2012.

7. R. Hadfield, "Single-photon detectors for optical quantum information applications," Nature Photonics $\mathbf{3}$, Dec. 2009.

8. Quantum Opus, Nanowire Datasheet, Jan. 2017.

9. A. J. Miller, A. E. Lita, B. Calkins, I. Vayshenker, S. M. Gruber, and S. W. Nam, "Compact cryogenic self-aligning fiber-to-detector coupling with losses below one percent," Optics Express 19, pp. 9102 - 9110, May 2011.

10. Quantum Opus, Nanowire Bias and Readout Electronics Datasheet, Jan. 2017.

11. V. B. Verma, B. Korzh, F. Bussières, R. D. Horansky, S. Dyer, A. Lita, L. Vayshenker, F. Marsili, M. D. Shaw, H. Zbinden, R. P. Mirin, and S. W. Nam, "High-efficiency superconducting nanowire single-photon detectors fabricated from MoSi thin-films," Optics Express 23, Dec. 2015.

12. F. Marsili, V. B. Verma, J. A. Stern, S. Harrington, A. E. Lita, T. Gerrits, I. Vayshenker, B. Baek, M. D. Shaw, R. P. Mirin, and S. W. Nam, "Detecting single infrared photons with $93 \%$ system efficiency," Nature Photonics 7, pp. 210 - 214, 2013.

13. S. Miki, M. Fujiwara, M. Sasaki, B. Baek, A. J. Miller, R. H. Hadfield, S. W. Nam, and Z. Weng, "Large sensitive-area $\mathrm{NbN}$ nanowire superconducting single-photon detectors fabricated on single-crystal MgO substrates," Applied Physics Letters 92, 2008.

14. B. Moison and J. Hamkins, "Coded modulation for the deep-space optical channel: Serially concatenated pulse position modulation," The Interplanetary Network Progress Report 42(161), 2005. 
15. J. M. Nappier and N. Lantz, "Development of an optical slice for an RF and optical software defined radio," in Proc. SPIE 10524 - Free-Space Laser Communication and Atmospheric Propagation XXX, 2018.

16. Consultative Committee for Space Data Systems (CCSDS), High Photon Efficiency Optical Communications: Coding \& Synchronization, Proposed Recommended Standard, Sep 2016.

17. T. A. Birks, I. Gris-Sánchez, S. Yerolatsitis, S. G. Leon-Saval, and R. R. Thomson, "The photonic lantern," Advances in Optics and Photonics 7, pp. $107-167,2015$. 